\title{
Angiotensin converting enzyme 2 activation: a novel potential Covid-19 therapeutic strategy
}

Haidy E. Michel

Department of Pharmacology and Toxicology, Faculty of Pharmacy, Ain Shams University, Cairo, Egypt

Corresponding author: Dr. Haidy E. Michel, Department of Pharmacology and Toxicology, Faculty of Pharmacy, Ain Shams University, Cairo, Egypt; E-mail: heidieffat@ pharma.asu.edu.eg.

\section{Competing interests}

The author declares no competing interests.

\section{Role of the funding source}

The present work did not receive any specific grant from funding agencies in the public, commercial, or not-for-profit sectors. 


\title{
Angiotensin converting enzyme 2 activation: a novel potential Covid-19 therapeutic strategy
}

\begin{abstract}
Severe acute respiratory syndrome coronavirus 2 (SARS-CoV-2) downregulates pulmonary ACE2 protein, resulting in pneumonia. Thus, increasing ACE2 activation may serve to both neutralize the virus and rescue cellular ACE2 activity protecting the lung from damage. Diminazene, an ACE2 activator, is suggested to be a novel tentative SARS-CoV-2 therapeutic.
\end{abstract}

Keywords: Covid-19; SARS-CoV-2; ACE2; Diminazene

\section{Background}

In late December 2019, the Covid-19 epidemic, caused by a novel coronavirus SARS-CoV-2, emerged in Wuhan, Hubei province, China. This epidemic has a doubling period of 1.8 days, and there are concerns about its progression to pandemic scales due to its exponential rate of spread ${ }^{1}$. No specific drugs or vaccines are currently available for the treatment and/or prevention of SARS-CoV-2 infection. Hence, there is an imperative need to search for a safe and effective therapeutic strategy for Covid-19 infected patients, especially the critically ill individuals.

\section{Covid-19 and ACE2}

Angiotensin-converting enzyme 2 (ACE2) is a crucial component of the renin-angiotensin-system (RAS) axis because it converts Angiotensin II into angiotensin (1-7), which exerts an antifibrotic, antihypertrophic and vasodilatory effect ${ }^{2}$. ACE2 is a membrane-bound aminopeptidase which has been reported to be a functional receptor for coronaviruses, including SARS-CoV and SARS-CoV-2 ${ }^{3}$. The first step of SARSCoV-2 infection is binding of the spike protein of the virus to ACE2 which is widely distributed on the alveolar type II cells and capillary endothelium ${ }^{4}$. It has been demonstrated that SARS-CoV downregulates ACE2 protein in mice, contributing to severe lung injury ${ }^{5}$. This suggests that augmented ACE2 activation 
may result in enhanced binding with SARS-CoV-2. Thus, increasing ACE2 activation may have a dual function to both neutralize the virus and rescue cellular ACE2 activity protecting the lung from damage.

\section{Diminazene as a potential Covid-19 therapeutic}

Diminazene (DIZE) is an antitripanosomal drug which has been shown to serve as an ACE2 activator and reduce bleomycin-induced pulmonary fibrosis ${ }^{6}$. In addition, it has been reported that activation of ACE2 by DIZE prevented asthma progression in rats by altering AKT, $\mathrm{p} 38, \mathrm{NF}-\kappa \mathrm{B}$ and other inflammatory markers ${ }^{7}$. DIZE also halted the development and progression of experimentally-induced pulmonary hypertension in rats, improved right ventricular function, and diminished proinflammatory cytokines effects that were accompanied with increased lung ACE2 activity ${ }^{8}$. Given the reported safety of DIZE administration in humans ${ }^{9,10}$ and the pressing need for Covid-19 therapeutic, in addition to the welldocumented pharmacological effects of DIZE, clinical studies are warranted to elucidate the potential safety and efficacy of DIZE in Covid-19 infected patients.

\section{Conclusion}

SARS-CoV-2 represents a global health challenge. Unfortunately, no specific therapeutic options are currently available. Thus, there is an imperative need for a safe and effective drug in order to put this pandemic to an end. DIZE has a reported acceptable safety profile. Moreover, DIZE increased lung ACE2 activity in different experimental models, an effect which conferred lung protection against various insults. Taking into consideration the reported effect of SARS-CoV-2 on pulmonary ACE2 activity, it could be suggested that DIZE administration could offer some therapeutic merit for SARS-CoV-2 infected patients. However, clinical studies are required to unravel the potential safety and efficacy of DIZE administration in Covid-19 infected patients. 


\section{References}

1. Cheng ZJ, Shan J. 2019 Novel coronavirus: where we are and what we know. Infection 2020: 1-9.

2. Zhou JP, Tang W, Feng Y, et al. Angiotensin-(1-7) decreases the expression of collagen I via TGF- $\beta 1 /$ Smad2/3 and subsequently inhibits fibroblast-myofibroblast transition. Clinical Science 2016; 130(21): 1983-91.

3. Turner AJ, Hiscox JA, Hooper NM. ACE2: from vasopeptidase to SARS virus receptor. Trends in pharmacological sciences 2004; 25(6): 291-4.

4. Lu R, Zhao X, Li J, et al. Genomic characterisation and epidemiology of 2019 novel coronavirus: implications for virus origins and receptor binding. The Lancet 2020; 395(10224): 565-74.

5. Kuba K, Imai Y, Rao S, et al. A crucial role of angiotensin converting enzyme 2 (ACE2) in SARS coronavirus-induced lung injury. Nature medicine 2005; 11(8): 875-9.

6. Shenoy V, Qi Y, Gupta D, Katovich M, Raizada M. Small moleculae ACE2 activator, diminazene aceturate attenuates bleomycin-induced pulmonary fibrosis. Eur Respiratory Soc; 2012.

7. Dhawale VS, Amara VR, Karpe PA, Malek V, Patel D, Tikoo K. Activation of angiotensinconverting enzyme 2 (ACE2) attenuates allergic airway inflammation in rat asthma model. Toxicology and applied pharmacology 2016; 306: 17-26.

8. Shenoy V, Gjymishka A, Jarajapu YP, et al. Diminazene attenuates pulmonary hypertension and improves angiogenic progenitor cell functions in experimental models. American journal of respiratory and critical care medicine 2013; 187(6): 648-57.

9. Pepin J, Milord F. The treatment of human African trypanosomiasis. Advances in parasitology 1994; 33: 1-47.

10. Hutchinson M, Watson H. Berenil in the treatment of Trypanosoma gambiense infection in man. Transactions of the Royal Society of Tropical Medicine and Hygiene 1962; 56(3): 227-30. 